\title{
Comparison of surgical difficulty in patients with resectable non-small cell lung cancer under different neoadjuvant treatment modes: a retrospective cohort study
}

\author{
Fan Zhang", Bin Qiü, Ying Ji, Wei Guo, Ning Li, Qi Xue, Shugeng Gao, Jie He \\ Department of Thoracic Surgery, National Cancer Center/National Clinical Research Center for Cancer/Cancer Hospital, Chinese Academy of \\ Medical Sciences and Peking Union Medical College, Beijing, China \\ Contributions: (I) Conception and design: S Gao, J He; (II) Administrative support: J He; (III) Provision of study materials or patients: W Guo; (IV) \\ Collection and assembly of data: F Zhang; (V) Data analysis and interpretation: B Qiu; (VI) Manuscript writing: All authors; (VII) Final approval of \\ manuscript: All authors. \\ \#These authors contributed equally to this work. \\ Correspondence to: Shugeng Gao. Department of Thoracic Surgery, National Cancer Center/National Clinical Research Center for Cancer/Cancer \\ Hospital, Chinese Academy of Medical Sciences and Peking Union Medical College, Panjiayuan Nanli 17, Chaoyang District, Beijing 100021, \\ China. Email: gaoshugeng@vip.sina.com.
}

Background: Previous studies have reported on the efficacy and safety of neoadjuvant use of a programmed cell death 1 (PD-1) antibody, sintilimab, in patients with non-small cell lung cancer (NSCLC). This study aimed to further evaluate the difficulty of this surgery and the postoperative complication rates in patients with NSCLC receiving neoadjuvant sintilimab.

Methods: Patients who received neoadjuvant sintilimab $(200 \mathrm{mg})$ in the Department of Thoracic Surgery, National Cancer Center/National Clinical Research Center for Cancer/Cancer Hospital from March 2018 to March 2019 were enrolled in the neoadjuvant immunotherapy group (NI group). Another two cohorts who did not receive sintilimab were retrospectively selected by propensity score matching (PSM) at a ratio of 1:1 in the upfront surgery (M-US) and neoadjuvant chemotherapy (M-NC) groups. The postoperative complication rate, postoperative days (PODs), and other detailed objective indicators were compared by $t$-test or $\chi^{2}$ test.

Results: Thirty-seven patients were enrolled in each group. Postoperative complications were greater in the NI group (37.8\%) than in the M-US $(10.8 \% ; \mathrm{P}=0.013)$ or in the M-NC group $(16.2 \% ; \mathrm{P}=0.036)$. The number of PODs (7) was greater in the NI group than in the M-US group ( $\mathrm{P}=0.005)$. The total number of dissected lymph nodes was lower in the NI group than in the M-US group $(\mathrm{P}<0.001)$ or in the M-NC group $(\mathrm{P}<0.001)$. Lymph node dissection $(\mathrm{LND})$ in the NI group was more difficult than in the M-US group $(\mathrm{P}=0.015)$, but intrathoracic adhesion, tumor invasion, and whole procedure difficulty were similar.

Conclusions: The administration of neoadjuvant sintilimab increased complications but did not increase the difficulty of surgery. Fewer lymph nodes were dissected in the NI group.

Keywords: Neoadjuvant immunotherapy; non-small cell lung cancer (NSCLC); subjective evaluation of surgery difficulty; postoperative complications; lymph node dissection (LND)

Submitted Jul 12, 2021. Accepted for publication Sep 10, 2021.

doi: $10.21037 /$ jtd-21-1007

View this article at: https://dx.doi.org/10.21037/jtd-21-1007 


\section{Introduction}

Lung cancer is one of the leading causes of morbidity and mortality worldwide (1). Non-small cell lung cancer (NSCLC) is the most common type of lung cancer that encompasses a heterogeneous class of tumors and represents around $85 \%$ of cases (2). Surgical treatment and radiochemotherapy play an important role in the prognosis of patients, particularly those with early stage NSCLC $(2,3)$. Meanwhile, targeted therapy for distinct molecular subtypes of NSCLC and immunotherapy has improved the treatment of patients with metastatic disease (4).

Immune checkpoint inhibitors (ICIs) have been widely applied in NSCLC in recent years (5). Neoadjuvant administration of ICIs in NSCLC has become popular worldwide since Forde et al. reported a high pathological response rate (6). Some patients with advanced NSCLC treated with one ICI type, an antibody against programmed cell death 1 (PD-1), have survived 5 years or longer (7). Application of ICIs before surgery has several advantages: (I) the vessels and lymph-vascular structures are intact, so pharmaceuticals can reach the tumor site and activate peripheral lymphocytes to destroy cancer cells (8). (II) Oncologists can obtain a pathological response to ICIs to guide postoperative treatment more individually. For example, patients with a major pathological response (MPR) may benefit from adjuvant use of the same ICI (9). (III) Preoperative cycle use of ICIs is often limited (4), so the financial burden for patients is mild compared to adjuvant therapy (often more than 12 cycles), which provides better patient compliance throughout the treatment procedure (10).

Despite the merits of neoadjuvant ICIs in NSCLC, concerns have also been raised for these blockades. Immune-related adverse events (irAEs) are a common cause of perioperative complications (i.e., pneumonitis, colitis, dermatitis, thyroiditis, hypophysitis), some of which are potentially lethal (11). In addition, surgeons are also concerned about the extra difficulty of tumor and lymph node resection due to ICI administration before surgery, which may include severe adhesions or fusion of lymph nodes stuck in the bifurcation of vessels (12). Most previous surgical studies of neoadjuvant ICIs were feasibility studies, reporting acceptable complication rates, little operation delay, and an optimal R0 resection rate. However, they rarely had controlled or matched groups (6,13-19).

Recently in our center, we evaluated the efficacy of a
PD-1 blockade (sintilimab) in neoadjuvant use for grade IA-IIIB NSCLC, which achieved a promising MPR of $40.5 \%(15 / 37)(19)$. This study aimed to investigate whether using sintilimab before surgery increases the difficulty of the procedure or increases the rate of complications. To do this, we evaluated the intraoperative and postoperative circumstances of a neoadjuvant ICI cohort and compared them to similar patients treated with preoperative chemotherapy and those treated with upfront surgery.

We present the following article in accordance with the STROBE reporting checklist (available at https://dx.doi. org/10.21037/jtd-21-1007).

\section{Methods}

\section{Patients}

In this cohort study, data from patients treated with neoadjuvant sintilimab were obtained from the phase $1 \mathrm{~b}$ trial for resectable NSCLC (Department of Thoracic Surgery, National Cancer Center/National Clinical Research Center for Cancer/Cancer Hospital, Chinese Academy of Medical Sciences, and Peking Union Medical College (registration No. ChiCTR-OIC-17013726) from March 2018 to March 2019. None of the received any other treatment before sintilimab therapy. The inclusion criteria were patients who were (I) 18-75 years old; (II) histologically or cytologically diagnosed with NSCLC (IA to IIIB, eighth edition of the American Joint Committee on Cancer) that could be surgically removed; (III) received sintilimab for the first time and had a diameter of the primary tumor greater than or equal to $2 \mathrm{~cm}$; (IV) with an Eastern Cooperative Oncology Group (ECOG) score of 0 ; and $(\mathrm{V})$ with adequate organ function. The exclusion criteria were patients who had (I) an epidermal growth factor receptor (EGFR)-sensitive gene mutation in tumor tissue; (II) any previous history of antitumor treatment, including radiotherapy, chemotherapy, immunotherapy, or traditional Chinese medicine; (III) received glucocorticoids within 4 weeks of the start of the study; (IV) suffered from known or suspected autoimmune diseases (including congenital or acquired), including interstitial pneumonia, uveitis, enteritis, hepatitis, pituitary inflammation, vasculitis, nephritis, or thyroiditis, or were currently suffering from interstitial lung disease; (V) received allogeneic transplantation (excluding corneal transplantation) or allogeneic hematopoietic stem cell transplantation; (VI) an allergy to any component of the monoclonal antibody 
treatment; (VII) suffered from other serious conditions that cannot be controlled, including but not limited to severe infection; HIV infection (HIV antibody positive); hepatitis $\mathrm{B}$ or acute or chronic active hepatitis $\mathrm{C}$, or active tuberculosis; III-IV congestive heart failure (New York Heart Association classification), arrhythmia, arterial hypertension, or other malignant tumors; (VIII) other acute or chronic diseases, mental illnesses, and women who were pregnant or breastfeeding. The study was conducted in accordance with the Declaration of Helsinki (as revised in 2013). The study was approved by ethics board of the Cancer Hospital, CAMS (No. 17-085/1340), and individual consent for this retrospective analysis was waived.

\section{Groups}

We retrospectively matched two cohorts to the ICI neoadjuvant cohort (NI group) from the clinical database of the Department of Thoracic Surgery using the propensity score matching (PSM) method to minimize selection bias. The characteristics selected for the match were clinical stage, sex, age at surgery, histological type, smoking history, and family history of cancer. All selected patients had an ECOG score of 0 . One cohort underwent upfront surgery without preoperative treatment (M-US group), and the other received platinum-based neoadjuvant chemotherapy (M-NC group). The clinical staging of all patients was determined by preoperative imaging examination with enhanced chest computed tomography (CT) or positron emission tomography (PET) CT, or pathology results from a biopsy of the primary tumor and suspected lymph nodes. Notably, we included only patients who underwent surgery at our center during the same period of the PD-1 blocking trial.

\section{Interventions}

Neoadjuvant ICI consisted of 2 cycles of intravenous sintilimab (200 mg/cycle) administered in patients with EGFR mutation-negative NSCLC (stage IA-IIIB, The American Joint Committee on Cancer/Union for International Cancer Control eighth version) before surgery with an interval of 3 weeks. Thirty-seven patients who received the PD-1 blockade agents and underwent surgery were included in this analysis of surgery-related complications (19). Patients in the NC group received platinum-based chemotherapy (pemetrexed + platinum or paclitaxel + platinum).

\section{Evaluation of surgical difficulty}

The total difficulty of surgery was evaluated according to the scoring for the extent of intrathoracic adhesion $(0=$ no adhesion; $1=$ mild/moderate; $2=$ severe), difficulty in lymph node dissection (LND) $(0=$ easy/normal; $1=$ hard; $2=$ extremely hard), tumor invasion $[0=$ no peripheral tissue invasion; $1=$ mild invasion of the lobar pulmonary artery $(\mathrm{PA}) /$ bronchus; $2=$ severe invasion of the main PA/ bronchus], and difficulty of the procedure as a whole $(0=$ easy/normal; $1=$ hard; 2 = extremely hard). Each indicator was classified into three grades according to the score given. Finally, each patient was classified according to the score.

\section{Observation indicators}

Objective indicators related to surgery were observed, including tumor locations, the extent of resection, surgical approach, duration of surgery, intraoperative blood loss, intrathoracic adhesions, postoperative complications, 30-day mortality, postoperative days (PODs) in hospital, postoperative drainage volume, resection margin, shrinkage of maximum tumor diameter, the maximum diameter of harvested lymph nodes, and the total number of harvested lymph nodes. Secondary outcomes were subjective variables, including the degree of intrathoracic adhesion, tumor invasion, the difficulty of LND, and difficulty of the entire operation procedure. All subjective variables were defined as ordered and categorical variables (i.e., for the degree of intrathoracic adhesion: $0=$ no adhesion; $1=$ mild adhesion; $2=$ massive/severe adhesion).

\section{Adverse events}

Documented postoperative complications included pneumonia, atelectasis, arrhythmia, prolonged air leak (more than 5 days), subcutaneous emphysema, chylothorax, hemorrhage, fever, and other irAEs, including hypophysitis and thyroiditis. All complications were classified according to the National Cancer Institute Common Terminology Criteria for Adverse Events version 5.0 (CTCAE 5.0).

\section{Data collection and follow-up}

The data for all patients were obtained from the electronic medical record system in the Department of Thoracic Surgery, National Cancer Center/National Clinical Research Center for Cancer/Cancer Hospital. All patients 


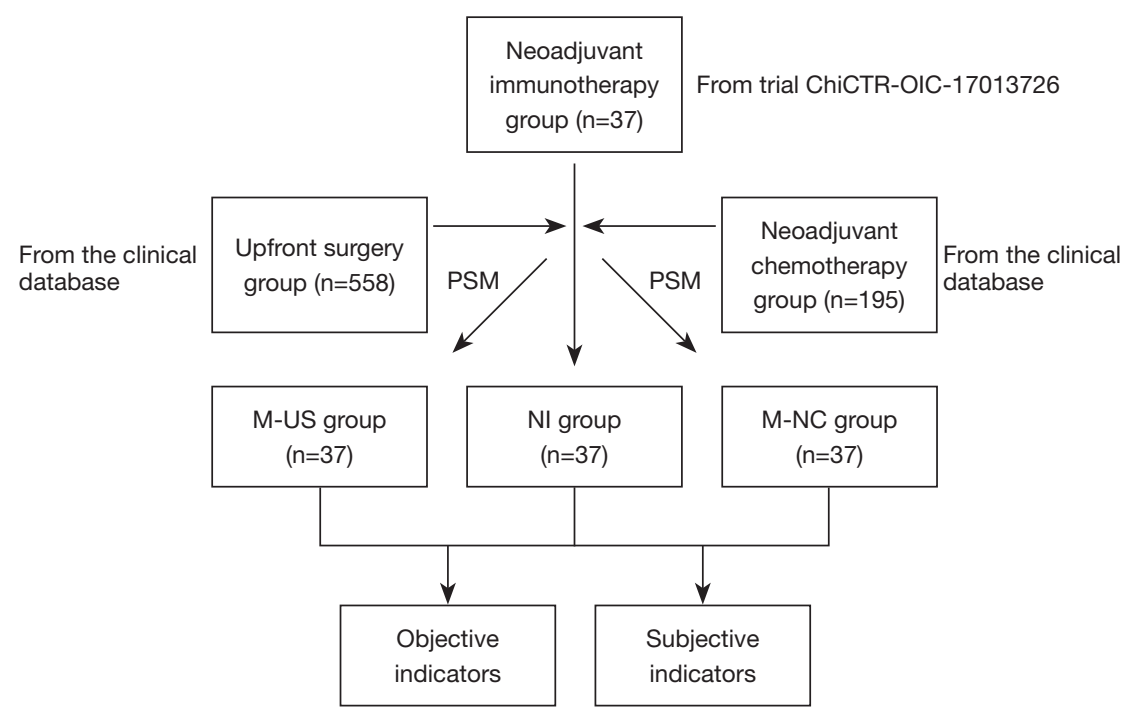

Figure 1 Flowchart of the study. The neoadjuvant immunotherapy group were from the phase $1 \mathrm{~b}$ trial of sintilimab treating resectable NSCLC (trial No. ChiCTR-OIC-17013726). The other two groups were matched cohorts from the clinical database of the Thoracic Surgery Department of our center. NI group: neoadjuvant immunotherapy group; M-US group: matched upfront surgery group; M-NC group: matched neoadjuvant chemotherapy group. NSCLC, non-small cell lung cancer; PSM, propensity score matching.

were followed up by telephone every 3 months.

\section{Statistical analysis}

All statistical analysis was performed using SPSS 22.0 for Windows (IBM Corp., Armonk, NY, USA). The Kolmogorov-Smirnov test was used to verify the normality of continuous data. Quantitative variables with a normal distribution (or nonnormal distribution) were described by mean \pm SD or median [range]. Quantitative variables with a normal distribution (or nonnormal distribution) between two groups were analyzed by Student's $t$-test (or MannWhitney $\mathrm{U}$ test). Categorical data are expressed as count or percentage (\%) and were analyzed by $\chi^{2}$ test (Pearson) and Fisher's exact test. The statistical significance level was set at a $P$ value $<0.05$.

\section{Results}

\section{Characteristics of unmatched cohorts and PSM}

From March 2018 to March 2019, 753 patients who underwent surgery at our center underwent chemotherapy, 558 of whom received upfront surgery (US group) and 195 of whom received neoadjuvant chemotherapy (NC group) (Figure 1). Additionally, 37 of 40 patients who received the PD-1 blockade agent underwent surgery (NI group). Among the remaining 3 patients, 2 patients had pleural metastases after thoracotomy and were switched to chemotherapy. In 1 case, surgical exploration indicated the patient could not be successfully resected, and this patient was switched to chemotherapy. Detailed characteristics of the US and NC groups before PSM with the NI group are listed in Table 1.

The proportion of women was significantly higher in the US group than in the NI group (38.0\% vs. $16.2 \%$; $\mathrm{P}=0.008$ ), while in the NC group, the proportion of women was only slightly higher than in the NI group $(28.2 \%$ vs. $16.2 \%$; $\mathrm{P}=0.129)$. Compared to the NI group, the mean age of the patients in the US and NC groups was not statistically significant $(\mathrm{P}>0.05)$. The proportion of squamous cell carcinoma cases was lower in the US group $(56.3 \%)$ and the NC group $(59.0 \%)$ than in the NI group $(83.8 \% ; \mathrm{P}=0.001$ and $\mathrm{P}=0.006$, respectively). The proportion of peripheral tumor, which was defined as the primary tumor that had not affected a segmental bronchus, was much higher in the US and NC group than in the NI group (77.4\% vs. $27.0 \%$, $\mathrm{P}<0.001 ; 50.3 \%$ vs. $27.0 \%, \mathrm{P}=0.009$, respectively). For the clinical stage $\mathrm{T}$, the proportion of $\mathrm{T} 1$ in the US group was higher than that in the NI group $(56.6 \%$ vs. $16.2 \%$; $\mathrm{P}<0.001$ ), while the proportion of $\mathrm{T} 1$ in the $\mathrm{NC}$ group was higher than that in the NI group $(39.0 \%$ vs. $16.2 \%$; 
Table 1 Patient characteristics of the three cohorts before matching (right) and after matching (left)

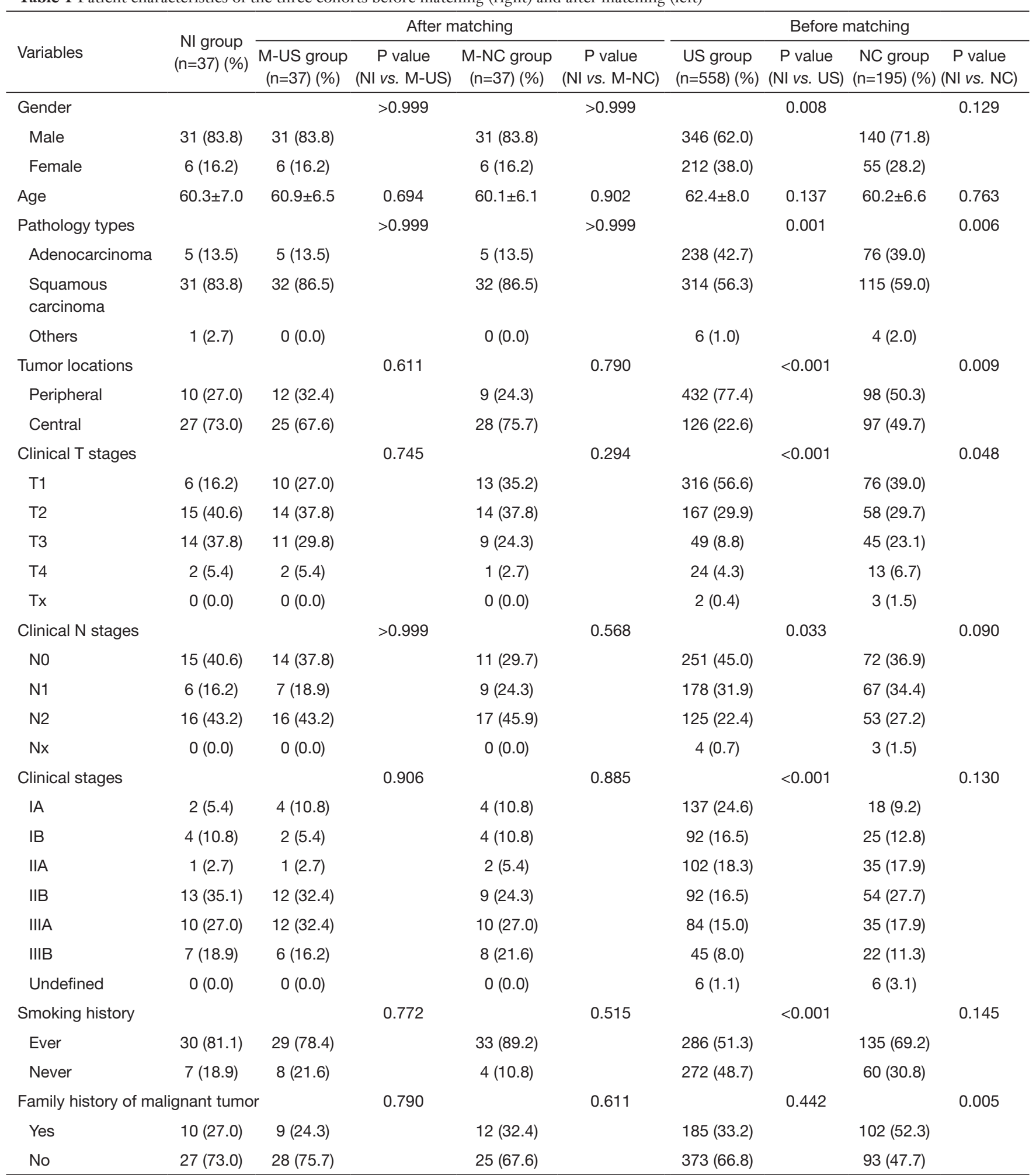

Quantitative variables with normal distribution are presented as mean \pm SD, and categorical data are presented as count or percentage (\%). The characteristics of PSM for matching include clinical stage, sex, age at surgery, histological type, smoking history, and family history of cancer. NI group: neoadjuvant immunotherapy group; M-US group: matched upfront surgery group; M-NC group: matched neoadjuvant chemotherapy group; US group: upfront surgery group; NC group: neoadjuvant chemotherapy group. PSM, propensity score matching. 
$\mathrm{P}=0.048$ ) but not significantly so. For the clinical stage $\mathrm{N}$, the proportion of $\mathrm{N} 2$ patients was lower in the US group than in the NI group ( $22.4 \%$ vs. $43.2 \% ; \mathrm{P}=0.033)$. For the total clinical stages, the proportion of patients in the cIA stage was significantly higher in the US group than in the NI group $(24.6 \%$ vs. $5.4 \% ; \mathrm{P}<0.001)$. The proportions for never-smokers and those with a family history of malignant tumors were significantly higher in the US group (48.7\% vs. $18.9 \% ; \mathrm{P}<0.001)$ and the $\mathrm{NC}$ group $(52.3 \%$ vs. $27.0 \%$; $\mathrm{P}=0.005$ ) than in the NI group, respectively.

As described above, this study used PSM and matched two cohorts (M-US group, $n=37$; $M-N C$ group, $n=37$ ) to minimize possible factors that may affect surgery difficulty. After matching, variables with significant differences before PSM were no longer significantly different between the groups (Table 1).

\section{Comparison of objective indicators related to surgery}

The distribution of tumor location (left $v s$. right, upper $v s$. middle $v s$. lower lobe) in the 3 groups was similar (Table 2). However, the proportion of tumors in the upper lobe was higher in the M-NC group than in the NI group but not significantly so $(70.3 \%$ vs. $45.9 \%$; $\mathrm{P}=0.097)$. Three groups were determined according to the extent of resection: lobectomy + (including lobectomy, lobectomy, plus wedge resection or segmentectomy of the adjacent lobe, and lobectomy, plus resection of the chest wall), combined/ sleeve lobectomy (including combined lobectomy including right upper lobe plus middle lobe, or the middle lobe plus the lower lobe, and sleeve resection), and pneumonectomy. The proportion of pneumonectomies was lower in the M-US group and the M-NC group than in the NI group, but not significantly so $(16.2 \%$ vs. $35.1 \%, \mathrm{P}=0.149 ; 27.0 \%$ vs. $35.1 \%, \mathrm{P}=0.264)$. Most surgeons in all three groups preferred open surgery to video-assisted thoracoscopic surgery (VATS). In all three groups, most the patients had R0 resection (36/37 in the NI group, $35 / 37$ in the $M-U S$ group, and $34 / 37$ in the M-NC group). The median duration of surgery in the M-NC group was longer than that in the NI group (140 vs. 109; $\mathrm{P}=0.011$ ), while the time in M-US and NI groups was the same (112 vs. 109; $\mathrm{P}=1.000)$. There were no significant differences in intraoperative blood loss between the NI and M-US groups (105 vs. 85; $\mathrm{P}=0.426$ ) or between the $\mathrm{NI}$ and $\mathrm{M}-\mathrm{NC}$ groups (105 vs. 120; $\mathrm{P}=0.098)$. The proportion of intrathoracic adhesions in the NI and M-US group $(\mathrm{P}=0.619)$ and in the $\mathrm{NI}$ and $\mathrm{M}-\mathrm{NC}$ group $(\mathrm{P}=0.806)$ was the same.
Patients in the NI group had a significantly higher complication rate for postoperative complications with clinical symptoms than did the other two groups (NI vs. M-US group: $37.8 \%$ vs. $10.8 \%, \mathrm{P}=0.013$; NI vs. $\mathrm{M}-\mathrm{NC}$ group: $37.8 \%$ vs. $16.2 \%, \mathrm{P}=0.036$; Table 2). Fifteen types of postoperative complications were documented in medical records. There were higher numbers in the NI group than in the other two groups in most types of complications, except for fever. Some complications were similar in the three groups (prolonged air leak, subcutaneous emphysema, chylothorax), while a minority of complications were more frequent in the two matched groups, including atelectasis and atrial fibrillation (Table 3). There was a minor difference in the number of complications cases above grade 3 (6 vs. 2 vs. 3; NI vs. US: $\mathrm{P}=0.261$; NI vs. NC: $\mathrm{P}=0.479$; Fisher's exact test). There were no deaths within 30 days after surgery in the M-US or M-NC groups, but two deaths occurred in the NI group $(\mathrm{P}=0.493)$. These two patients almost covered all severe complications, including altered consciousness, hyponatremia, pneumonia, diabetes insipidus, and fever, as described in an earlier report (19).

There were no differences in the max diameter of the harvested lymph nodes in the NI and M-US groups $(\mathrm{P}=0.350)$ or the $\mathrm{NI}$ and $\mathrm{M}-\mathrm{NC}$ groups $(\mathrm{P}=0.323)$. Surgeons removed fewer lymph nodes in the NI group than in the M-US or M-NC groups $(\mathrm{P}<0.001)$. The median number of days after surgery in the hospital (POD) in the NI group was 7 days, which was greater than that in the M-US group $(\mathrm{P}=0.005)$ and the same as that in the $\mathrm{M}-\mathrm{NC}$ group $(\mathrm{P}=0.987)$. The postoperative total drainage volume of the NI group was higher than that of the M-US group $(\mathrm{P}=0.193)$ but lower than that of the $\mathrm{M}-\mathrm{NC}$ group $(\mathrm{P}=0.439)$; however, neither difference was significant (Table 2).

\section{Evaluation of operation difficulty}

For the proportion of intrathoracic adhesions, there was almost no difference between the NI and M-US groups $(\mathrm{P}=0.920)$ or between the $\mathrm{NI}$ and $\mathrm{M}-\mathrm{NC}$ groups $(\mathrm{P}>0.999)$. For LND, the proportion patients with easy/ normal grade in the NI group was significantly higher than that in the M-US group $(\mathrm{P}=0.015)$, but not significantly different to that of the M-NC group $(\mathrm{P}=0.409)$. There was no difference in the extent of tumor invasion to the $\mathrm{PA}$ and bronchus in the NI and the M-US groups or the $\mathrm{NI}$ and the M-NC groups. For the surgical procedure as a whole, $56.8 \%$ of surgeons considered it easy or normal in the NI group, while the proportion in the M-US and 
Table 2 Comparison of objective indicators in the different groups

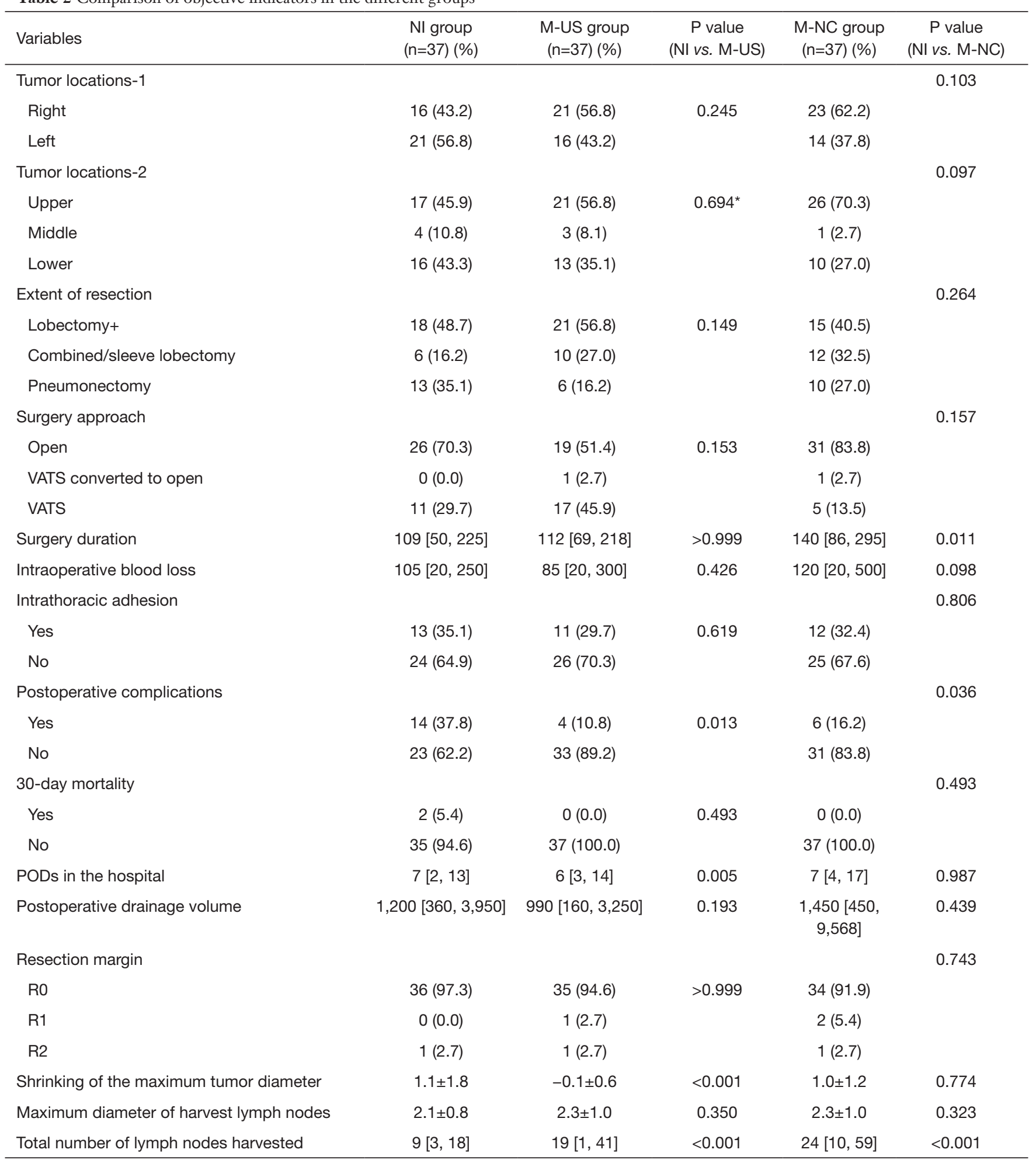

Quantitative variables with a normal distribution (or nonnormal distribution) are presented a mean \pm SD or median [range], and categorical data are presented as count or percentage (\%). *, Fisher's exact test. NI group: neoadjuvant immunotherapy group; M-US group: matched upfront surgery group; M-NC group: matched neoadjuvant chemotherapy group. VATS, video-assisted thoracoscopic surgery; PODs, postoperative days. 
Table 3 Detailed complications after surgery in the three groups

\begin{tabular}{|c|c|c|c|c|c|c|}
\hline Variables & \multicolumn{2}{|c|}{$\mathrm{NI}$ group $(\mathrm{n}=37)$} & \multicolumn{2}{|c|}{ M-US group $(n=37)$} & \multicolumn{2}{|c|}{ M-NC group $(n=37)$} \\
\hline Prolonged air leak & $1(2.7)$ & $0(0.0)$ & $1(2.7)$ & $1(2.7)$ & $2(5.4)$ & $0(0.0)$ \\
\hline Subcutaneous emphysema & $0(0.0)$ & $0(0.0)$ & $0(0.0)$ & $0(0.0)$ & $1(2.7)$ & $1(2.7)$ \\
\hline Hemoptysis & $2(5.4)$ & $0(0.0)$ & $0(0.0)$ & $0(0.0)$ & $0(0.0)$ & $0(0.0)$ \\
\hline Intrathoracic bleeding & $3(8.1)$ & $2(5.4)$ & $1(2.7)$ & $1(2.7)$ & $0(0.0)$ & $0(0.0)$ \\
\hline Subcutaneous ecchymosis & $1(2.7)$ & $0(0.0)$ & $0(0.0)$ & $0(0.0)$ & $0(0.0)$ & $0(0.0)$ \\
\hline Chylothorax & $1(2.7)$ & $0(0.0)$ & $0(0.0)$ & $0(0.0)$ & $1(2.7)$ & $1(2.7)$ \\
\hline Pneumonia & $2(5.4)$ & $2(5.4)$ & $0(0.0)$ & $0(0.0)$ & $0(0.0)$ & $0(0.0)$ \\
\hline Diabetes insipidus & $1(2.7)$ & $1(2.7)$ & $0(0.0)$ & $0(0.0)$ & $0(0.0)$ & $0(0.0)$ \\
\hline Hyponatremia & $1(2.7)$ & $1(2.7)$ & $0(0.0)$ & $0(0.0)$ & $0(0.0)$ & $0(0.0)$ \\
\hline Incision infection & $1(2.7)$ & $1(2.7)$ & $0(0.0)$ & $0(0.0)$ & $0(0.0)$ & $0(0.0)$ \\
\hline High blood pressure & $2(5.4)$ & $0(0.0)$ & $0(0.0)$ & $0(0.0)$ & $1(2.7)$ & $0(0.0)$ \\
\hline Atrial fibrillation & $0(0.0)$ & $0(0.0)$ & $0(0.0)$ & $0(0.0)$ & $2(5.4)$ & $1(2.7)$ \\
\hline Total (events) & $22(59.5)$ & $10(27.0)$ & $6(16.2)$ & $2(5.4)$ & $9(24.3)$ & $3(8.1)$ \\
\hline Total (patients) & $14(37.8)$ & $6(16.2)$ & $4(10.8)$ & $2(5.4)$ & $6(16.2)$ & $3(8.1)$ \\
\hline
\end{tabular}

Categorical data are presented as count or percentage (\%). All complications are classified according to CTCAE 5.0. NI group: neoadjuvant immunotherapy group; M-US group: matched upfront surgery group; M-NC group: matched neoadjuvant chemotherapy group. CTCAE 5.0, Common Terminology Criteria for Adverse Events version 5.0.

M-NC groups was $59.5 \%(\mathrm{P}=0.777)$ and $48.6 \%(\mathrm{P}=0.800)$, respectively. The proportion of $\mathrm{T}$ or $\mathrm{N}$ downstaging in the NI group was higher than that in the M-NC group, but not significantly so ( $\mathrm{T}$ downstaging: $43.2 \%$ vs. $29.7 \%, \mathrm{P}=0.227$; $\mathrm{N}$ downstaging: $29.7 \%$ vs. $16.2 \%, \mathrm{P}=0.167$; Table 4).

\section{Discussion}

This study evaluated the difficulty of surgery and the postoperative complications rates of NSCLC patients treated with neoadjuvant sintilimab. Surgical factors and complications were compared in the NI group with two other PSM-matched cohorts of PSM-matched patients with NSCLC who underwent surgery. The results showed that postoperative complications were higher in the NI group than in the M-US and M-NC groups. The number of PODs in the NI group was greater than that in the M-US group, and there were fewer dissected lymph nodes in the NI group than in the other groups. Dissection of the lymph nodes in the NI group was more difficult than in the M-US group, but intrathoracic adhesion, tumor invasion, and whole procedure difficulty were similar. This suggests that neoadjuvant sintilimab administration increased complications but did not increase the difficulty of surgery. The reason why fewer lymph nodes were dissected in patients in the NI group warrants further investigation.

The presence of more complications in the NI group contradicts some of the findings of previous research. In most stage I-II clinical trials for the neoadjuvant administration of ICIs in NSCLC that have shown promising MPR rates, the researchers found that ICIs before surgery did not increase the rate of postoperative complications, R1/R2 margin, or inoperable cases (13-19). However, the reference standard for the postoperative complication rate was previous studies, including the SWOG-S9900, ANITA, JBR-10, and IALT (15-23) trials, 
Table 4 Comparison of surgical difficulty in the different groups

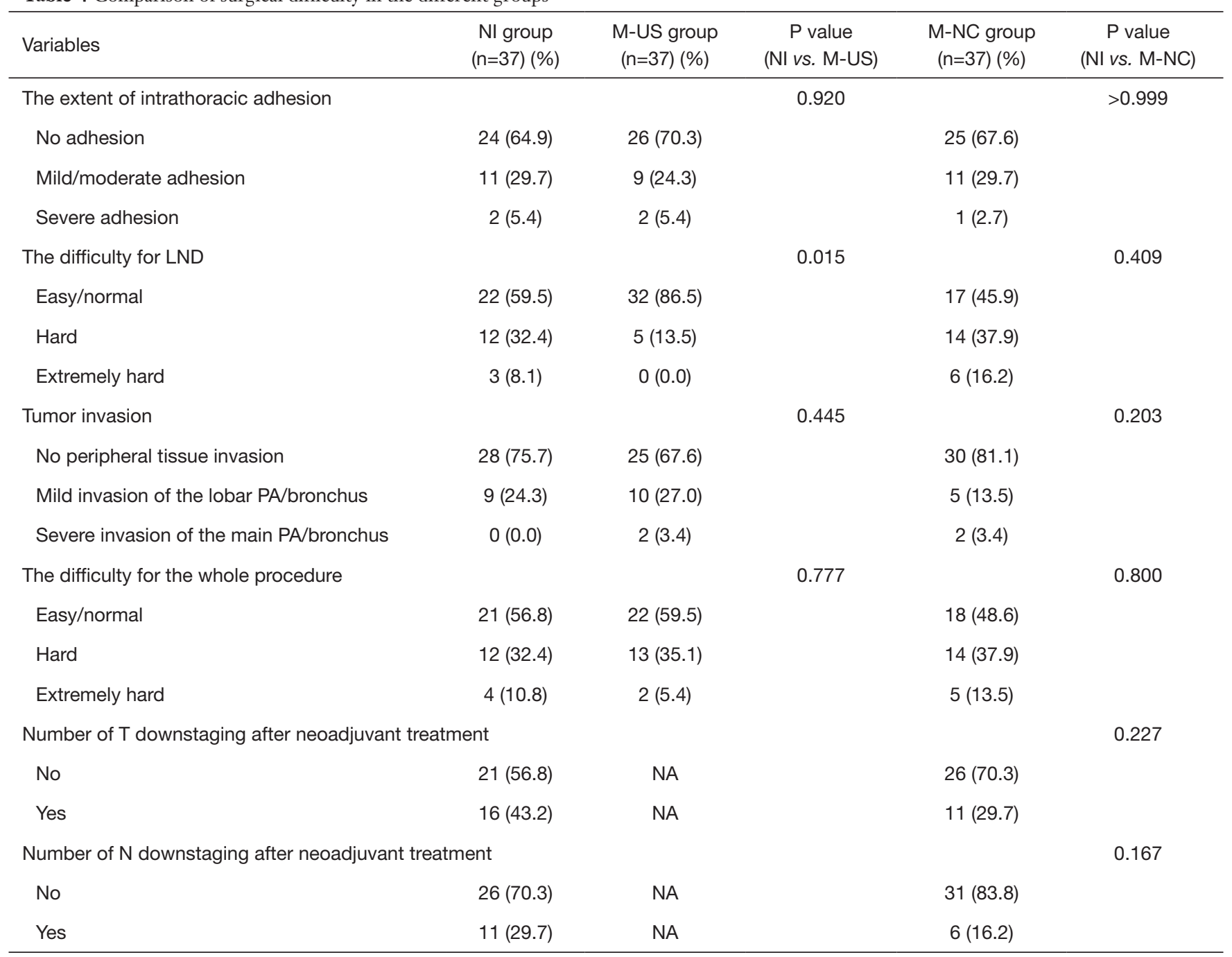

Categorical data are presented as count or percentage (\%). NI group: neoadjuvant immunotherapy group; M-US group: matched upfront surgery group; M-NC group: matched neoadjuvant chemotherapy group. LND, lymph node dissection; PA, pulmonary artery.

in which clinical stages, tumor locations, and other factors that might be related to complications were not comparable with the neoadjuvant immunotherapy group. In this study, two cohorts from the same medical center were matched in the same treatment period by PSM to minimize possible confounders. The postoperative complications rate (37.8\%) was significantly higher in the NI group than in the other two groups (10.8\% and $16.2 \%)$. The absolute complication rate was comparable with that of other studies $(6,17,18)$ at approximately $30 \%$, and most were mild (grade 1 or 2 ). However, the complications in the two matched groups were much lower. Previous studies have reported rates of up to $50 \%$ for complications after pulmonary surgery with neoadjuvant chemotherapy. The level of $10-20 \%$ in this study was consistent with the common clinical experience in our center (24) and China (25) perhaps for the following reasons: (I) more than 10 years' (2018-2019 vs. 2000-2006) difference between the treatment periods allowed centers to become more skilled in operations; (II) the retrospective matching of neoadjuvant chemotherapy patients could have caused selection bias in which only patients who could tolerate chemotherapy drugs were enrolled in the cohort; and (III) the limited numbers in the cohorts might have led to some less common complications being missed.

However, the rate of complication for the NI group should not be neglected. There were more severe 
complications, including ICI-induced pneumonia, disturbance of consciousness, and intrathoracic bleeding in the NI group than in the other two groups, but these differences were not significant. Two cases died within 30 days after surgery, one due to ICI-related pneumonia and one for sudden high fever, electrolyte disturbance, and diabetes insipidus. This was possibly due to hypophysitis, indicated by moderate elevated 2-[fluorine-18]-fluoro2-deoxy-D-glucose (FDG)-avidity in PET-CT, after ICI use as we previously reported (19). The number of PODs was also greater in the NI group than in the M-US group, which was related to complications. One possible reason for the increase in complications is that the internal environment alterations caused by ICI use could be more severe than those of chemotherapy, leading to variations in inflammatory and coagulation factors (26). Our previous studies have shown that sintilimab rarely causes common adverse effects such as those encountered with platinum-based chemotherapy, such as vomiting, alopecia, or myelosuppression (19). However, the results of this study are a reminder that ICIs are not as mild as they may appear and activated immune cells can give patients additional susceptibility to an unpredictable crisis. During the postoperative period, life-threatening complications, including pneumonia, bleeding, and electrolyte disturbance, had to be meticulously managed and treated.

There were few differences in most intraoperative indicators, including surgical approach, intraoperative blood loss, intrathoracic adhesion, or tumor invasion. Most importantly, the duration of surgery (median 109 minutes) of the NI group was not longer than that of the other two groups, indicating that the difficulty of the surgery was under control after the ICI was infused, which was consistent with the subjective evaluation of the difficulty of the surgery indicating that the proportion of difficult or extremely hard surgery was about $40-50 \%$ for three groups. Many surgeons were concerned about the additional difficulties they might encounter due to the action of activated immune cells on cancer cells, and there have been no previous studies that have reported a subjective evaluation of surgery difficulty after ICIs. The results of this study could offer reasonable confidence for surgeons in using sintilimab before surgery.

Another interesting finding was the difference in the number of lymph nodes harvested between the NI group and the other two groups. The median number of lymph nodes harvested was only half of the other two groups (9 vs. 19 vs. 24). As surgeons, we all understand the importance of LND in thoracic surgery, especially for these cohorts of patients, most of whom were in the N1-2 stage. Some retrospective studies have shown that the number of lymph nodes harvested was significantly associated with the overall survival of the patients (27-29). An obvious reason for the difference of harvested number of lymph nodes is the difficulty. The proportion of LND subjectively evaluated as hard/extremely hard in the NI group was significantly higher than that in the M-US group (40.5\% vs. $13.5 \%$ ), indicating the elevated difficulties caused by ICI administration. However, the proportion of difficult LND was high in the M-NC group $(54.1 \%)$, and in that group, it was not related to fewer lymph nodes harvested (median number 24). If LND was hampered by severe adhesion or invasion between lymph nodes and vessels or other surrounding tissues, the harvested lymph nodes should be smaller and more fragmented, and the maximum diameter should decrease, but we did not observe this (mean value 2.1 vs. 2.3 vs. 2.3; $\mathrm{P}>0.05$ ). In recent research, Liang et al. found fewer lymph nodes in an immunotherapy + chemotherapy group than in a chemotherapy group, up to $51 \%$ at the N2 station. They attributed the decrease in lymph node number to difficult dissection after the administration of PD-1 blockade (30). Another probable reason for the fewer lymph nodes harvested in the NI group was that sintilimab could cause dramatic changes in the lymph nodes, such as fusion or shrinking. However, if so, the size of the lymph nodes in the NI group could be larger than that in the other two groups. Previous studies showed that PD-1 blockade significantly affected the tumor's microenvironment, favoring $\mathrm{CD}^{+} \mathrm{T}$ cell infiltration and therefore improved anticancer efficacy (31). Whether ICIs affect lymph nodes at the macrolevel needs to be further investigated.

The duration of the surgery was also affected by the difference in the difficulty of dissection of the lymph nodes in the three groups. A pulmonary surgery (lobectomy or pneumonectomy) ( $\mathrm{T}$ ) comprises three parts: separation of adhesion $\left(\mathrm{T}_{\mathrm{a}}\right)$, resection of the lobe(s) $\left(\mathrm{T}_{1}\right)$, and dissection of lymph nodes $\left(T_{n}\right)$. Let $D_{n}$ represent the difficulty in the dissection of the lymph nodes and $\mathrm{N}$ represent the number of lymph nodes harvested. As the adhesion and difficulty of lobe(s) resection were the same in three groups, we determined $T_{a}$ and $T_{1}$ to be equal in the three groups. Therefore, the duration of surgery for the entire procedure depended on $T_{n}$. Theoretically, $T_{n} \propto D_{n} \times N$. In the case of the NI and M-US groups, $\mathrm{D}_{\mathrm{n}-\mathrm{NI}}>\mathrm{D}_{\mathrm{n}-\mathrm{M}-\mathrm{US}}$ and $\mathrm{N}_{\mathrm{NI}}<\mathrm{N}_{\mathrm{M}-\mathrm{US}}$, so $\mathrm{T}_{\mathrm{n}-\mathrm{NI}} \approx \mathrm{T}_{\mathrm{n} \text {-M-Us }}$, and thus $\mathrm{T}_{\mathrm{NI}} \approx \mathrm{T}_{\mathrm{M} \text {-Us }}$. In the case of the 
$\mathrm{NI}$ and M-NC groups, $\mathrm{D}_{\mathrm{n}-\mathrm{NI}} \approx \mathrm{D}_{\mathrm{n}-\mathrm{M}-\mathrm{NC}}$ and $\mathrm{N}_{\mathrm{NI}}<\mathrm{N}_{\mathrm{M}-\mathrm{NC}}$, so

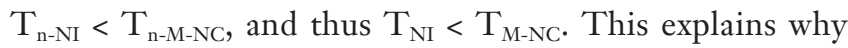
the surgical duration was significantly longer in the M-NC group.

By imaging observation, we also noticed that the shrinkage of the maximum tumor diameter in the NI group was generally the same as that in the M-NC group (1.1 \pm 1.8 vs. $1.0 \pm 1.2 ; \mathrm{P}=0.774)$, indicating that ICI administration did not have an advantage in decreasing tumor diameter over chemotherapy. In this study, 9 of the 37 patients had a severe response. Theoretically, ICIs may recruit more immune cells to the tumor site so that more "swelling" of the tumor occurs than that induced by chemotherapeutic or targeted kinase inhibitor drugs that directly eliminate tumor cells. Thus, an efficacy evaluation indicator based on imaging examination, including objective response rate (ORR), may not represent the "real" treatment effect of ICIs like sintilimab. Administration of ICIs before surgery plays an irreplaceable role in guiding subsequent treatment options because it can lead to a pathological response.

There were some limitations in this study. First, the sample size was limited due to the stage I-II nature of the neoadjuvant sintilimab trial, making the statistical test result more vulnerable to random errors. The second limitation was the retrospective nature of the study. Although PSM selected two groups to minimize the factors that might have affected the difficulty of surgery, the baseline condition of the three groups of patients may still differ in unknown ways. Third, the subjective evaluation of surgical difficulty was extremely challenging to quantify. We tried to invent a scoring system for the subjective evaluation of surgery difficulty and finally used categorical variables that only contained a few ordered values (i.e., $0=$ easy; $1=$ hard; $2=$ extremely hard) to account for differences in the sensory degree of different personnel. This might have led to different results compared to other studies.

\section{Conclusions}

This study was the first to report the increase in complications due to neoadjuvant use of sintilimab in NSCLC according to two well-matched cohorts that were comparable to the neoadjuvant ICI group in the clinical stage and treatment period. This study also found that fewer lymph nodes were harvested in the immunotherapy group than in the initial surgery group or the neoadjuvant chemotherapy group; the underlying reasons for this need to be investigated further.

\section{Acknowledgments}

We thank Dr. Yushun Gao, Kang Shao, Xinjie Chen, Fang Lv, Ruochuan Zang, and Shuai Cui for their hard work. Funding: This work was supported by the National Key Research Development Plan (No. 2021YFC250009), the CAMS Initiative for Innovative Medicine (CAMS-I2M; No. 2017-I2M-2-003), and the Beijing Hope Run Special Fund of the Cancer Foundation of China (No. LC2017B20).

\section{Footnote}

Reporting Checklist: The authors have completed the STROBE reporting checklist. Available at https://dx.doi. org/10.21037/jtd-21-1007

Data Sharing Statement: Available at https://dx.doi. org/10.21037/jtd-21-1007

Conflicts of Interest: All authors have completed the ICMJE uniform disclosure form (available at https://dx.doi. org/10.21037/jtd-21-1007). The authors have no conflicts of interest to declare.

Ethical Statement: The authors are accountable for all aspects of the work in ensuring that questions related to the accuracy or integrity of any part of the work are appropriately investigated and resolved. The study was conducted in accordance with the Declaration of Helsinki (as revised in 2013). The study was approved by ethics board of the Cancer Hospital, CAMS (No. 17-085/1340), and individual consent for this retrospective analysis was waived.

Open Access Statement: This is an Open Access article distributed in accordance with the Creative Commons Attribution-NonCommercial-NoDerivs 4.0 International License (CC BY-NC-ND 4.0), which permits the noncommercial replication and distribution of the article with the strict proviso that no changes or edits are made and the original work is properly cited (including links to both the formal publication through the relevant DOI and the license). See: https://creativecommons.org/licenses/by-nc-nd/4.0/.

\section{References}

1. Bade BC, Dela Cruz CS. Lung cancer 2020: epidemiology, 
etiology, and prevention. Clin Chest Med 2020;41:1-24.

2. Gridelli C, Rossi A, Carbone DP, et al. Non-small-cell lung cancer. Nat Rev Dis Primers 2015;1:15009.

3. Ettinger DS, Wood DE, Akerley W, et al. NCCN guidelines insights: non-small cell lung cancer, version 4.2016. J Natl Compr Canc Netw 2016;14:255-64.

4. Shroff GS, de Groot PM, Papadimitrakopoulou VA, et al. Targeted therapy and immunotherapy in the treatment of non-small cell lung cancer. Radiol Clin North Am 2018;56:485-95.

5. Broderick SR, Bott MJ. Neoadjuvant immunotherapy in patients with resectable non-small cell lung cancer. J Thorac Cardiovasc Surg 2019;158:1471-4.

6. Forde PM, Chaft JE, Smith KN, et al. Neoadjuvant PD-1 Blockade in Resectable Lung Cancer. N Engl J Med 2018;378:1976-86. Erratum in: N Engl J Med 2018;379:2185.

7. Yoneda K, Imanishi N, Ichiki Y, et al. Immune checkpoint inhibitors (ICIs) in non-small cell lung cancer (NSCLC). J UOEH 2018;40:173-89.

8. Liu J, Blake SJ, Yong MC, et al. Improved efficacy of neoadjuvant compared to adjuvant immunotherapy to eradicate metastatic disease. Cancer Discov 2016;6:1382-99.

9. Hellmann MD, Chaft JE, William WN Jr, et al. Pathological response after neoadjuvant chemotherapy in resectable non-small-cell lung cancers: proposal for the use of major pathological response as a surrogate endpoint. Lancet Oncol 2014;15:e42-50.

10. Jia XH, Xu H, Geng LY, et al. Efficacy and safety of neoadjuvant immunotherapy in resectable nonsmall cell lung cancer: a meta-analysis. Lung Cancer 2020;147:143-53.

11. Michot JM, Bigenwald C, Champiat S, et al. Immunerelated adverse events with immune checkpoint blockade: a comprehensive review. Eur J Cancer 2016;54:139-48.

12. O'Donnell JS, Hoefsmit EP, Smyth MJ, et al. The promise of neoadjuvant immunotherapy and surgery for cancer treatment. Clin Cancer Res 2019;25:5743-51.

13. Kwiatkowski DJ, Rusch VW, Chaft JE, et al. Neoadjuvant atezolizumab in resectable non-small cell lung cancer (NSCLC): interim analysis and biomarker data from a multicenter study (LCMC3). J Clin Oncol 2019;37:abstr 8503.

14. Cascone T, William WN, Weissferdt A, et al. Neoadjuvant nivolumab $(\mathrm{N})$ or nivolumab plus ipilimumab (NI) for resectable non-small cell lung cancer (NSCLC): clinical and correlative results from the NEOSTAR study. J Clin Oncol 2019;37:abstr 8504.
15. Provencio M, Nadal E, Insa A, et al. Neoadjuvant chemo-immunotherapy for the treatment of stage IIIA resectable non-small-cell lung cancer (NSCLC): a phase II multicenter exploratory study-final data of patients who underwent surgical assessment. J Clin Oncol 2019;37:abstr 8509 .

16. Bar J, Urban D, Ofek E, et al. Neoadjuvant pembrolizumab (Pembro) for early stage non-small cell lung cancer (NSCLC): updated report of a phase I study, MK3475-223. J Clin Oncol 2019;37:abstr 8534.

17. Bott MJ, Cools-Lartigue J, Tan KS, et al. Safety and feasibility of lung resection after immunotherapy for metastatic or unresectable tumors. Ann Thorac Surg 2018;106:178-83.

18. Bott MJ, Yang SC, Park BJ, et al. Initial results of pulmonary resection after neoadjuvant nivolumab in patients with resectable non-small cell lung cancer. J Thorac Cardiovasc Surg 2019;158:269-76.

19. Gao S, Li N, Gao S, et al. Neoadjuvant PD-1 inhibitor (Sintilimab) in NSCLC. J Thorac Oncol 2020;15:816-26.

20. Pisters KM, Vallières E, Crowley JJ, et al. Surgery with or without preoperative paclitaxel and carboplatin in earlystage non-small-cell lung cancer: Southwest Oncology Group Trial S9900, an intergroup, randomized, phase III trial. J Clin Oncol 2010;28:1843-9.

21. Douillard JY, Rosell R, De Lena M, et al. Adjuvant vinorelbine plus cisplatin versus observation in patients with completely resected stage IB-IIIA non-small-cell lung cancer (Adjuvant Navelbine International Trialist Association ANITA): a randomised controlled trial. Lancet Oncol 2006;7:719-27.

22. Butts CA, Ding K, Seymour L, et al. Randomized phase III trial of vinorelbine plus cisplatin compared with observation in completely resected stage IB and II nonsmall-cell lung cancer: updated survival analysis of JBR-10. J Clin Oncol 2010;28:29-34.

23. Dunant A, Pignon JP, Le Chevalier T. Adjuvant chemotherapy for non-small cell lung cancer: contribution of the International Adjuvant Lung Trial. Clin Cancer Res 2005;11:5017s-21s.

24. Zang R, Shi JF, Lerut TE, et al. Ten-year trends of clinicopathologic features and surgical treatment of lung cancer in China. Ann Thorac Surg 2020;109:389-95.

25. Mei J, Guo C, Xia L, et al. Long-term survival outcomes of video-assisted thoracic surgery lobectomy for stage I-II non-small cell lung cancer are more favorable than thoracotomy: a propensity score-matched analysis from a high-volume center in China. Transl Lung Cancer Res 
2019;8:155-66.

26. Sato R, Imamura K, Sakata S, et al. Disorder of coagulation-fibrinolysis system: an emerging toxicity of anti-PD-1/PD-L1 monoclonal antibodies. J Clin Med 2019;8:762.

27. Liang W, He J, Shen $Y$, et al. Impact of examined lymph node count on precise staging and long-term survival of resected non-small-cell lung cancer: a population study of the US SEER database and a Chinese multi-institutional registry. J Clin Oncol 2017;35:1162-70.

28. Yendamuri S, Dhillon SS, Groman A, et al. Effect of the number of lymph nodes examined on the survival of patients with stage I non-small cell lung cancer who undergo sublobar resection. J Thorac Cardiovasc Surg 2018;156:394-402.

29. Saji H, Tsuboi M, Yoshida K, et al. Prognostic impact of number of resected and involved lymph nodes at complete resection on survival in non-small cell lung cancer. J Thorac Oncol 2011;6:1865-71.

30. Liang H, Yang C, Gonzalez-Rivas D, et al. Sleeve lobectomy after neoadjuvant chemoimmunotherapy/ chemotherapy for local advanced non-small cell lung cancer. Transl Lung Cancer Res 2021;10:143-55.

31. Fransen MF, Schoonderwoerd M, Knopf P, et al. Tumordraining lymph nodes are pivotal in PD-1/PD-L1 checkpoint therapy. JCI Insight 2018;3:124507.

Cite this article as: Zhang F, Qiu B, Ji Y, Guo W, Li N, Xue Q, Gao S, He J. Comparison of surgical difficulty in patients with resectable non-small cell lung cancer under different neoadjuvant treatment modes: a retrospective cohort study. J Thorac Dis 202 1;13(10):5604-5616. doi: 10.21037/jtd-21-1007 